\title{
Classifications of vitreomacular traction syndrome: diameter vs morphology
}

J Bottós ${ }^{1}$, J Elizalde², EB Rodrigues', M Farah and M Maia ${ }^{1}$

\begin{abstract}
Purpose The aim of this study is to analyze the agreement between the classifications based on morphology and diameter of vitreomacular traction (VMT) syndrome, as well as to correlate the morphological findings of VMT with specific maculopathies. Methods Fifty-three eyes with VMT syndrome were categorized into two classifications based on optical coherence tomography images: the VMT morphology (V- or J-shaped) and the diameter of adhesion (focal $\leq 1500 \mu \mathrm{m}$ or broad $>1500 \mu \mathrm{m}$ ).

Results High correlation was seen between V-shaped and focal-VMT and between $\mathrm{J}$-shaped and broad-VMT (kappa $=0.850$; $P<0.001$ ), except in four cases with broad adhesion despite the presence of a V-shaped pattern. These four cases had common characteristics to those with broad vitreal attachment regarding associated maculopathies and visual function. V-shaped VMT $(n=29)$ and focal-VMT $(n=25)$ led to tractional cystoid macular edema (CME; $79.31 \%$ and $84 \%$, respectively) and macular hole (MH; 37.93\% and 44\%); J-shaped VMT $(n=24)$ and broad-VMT $(n=28)$ were associated with epiretinal membranes (ERMs; $91.66 \%$ and $92.85 \%$, respectively) and diffuse retinal thickening $(62.50 \%$ and $64.28 \%)$. The best-corrected visual acuity (BCVA) was not significantly different between the groups (BCVA logarithm of the minimum angle of resolution: V-shaped, 0.45; J-shaped, 0.46; $P=0.816$; and focal, 0.50 ; broad, $0.42 ; P=0.198$ ). Conclusions Although highly concordant, the classification based on the diameter of the adhesion and not on the classical adhesion morphology seemed to better reflect the specific macular changes. V-shaped and focal VMT led to tractional CME and MH, while J-shaped and broad VMT were
\end{abstract}

associated with ERM and diffuse retinal thickening.

Eye (2014) 28, 1107-1112; doi:10.1038/eye.2014.128; published online 4 July 2014

\section{Introduction}

The advent of a new method of high-definition optical coherence tomography (HD-OCT) has provided new insight into vitreomacular traction (VMT) syndrome through improved evaluation of the tractional forces at the vitreoretinal interface ${ }^{1-3}$ and the relationship of these tractional forces with specific macular conditions. ${ }^{4}$ In the past, VMT syndrome was considered an isolated pathology. Currently, it is believed to contribute to a wide spectrum of macular diseases, including cystoid macular edema (CME), macular holes (MHs) and epiretinal membranes (ERMs). ${ }^{4}$ Although the pathogeneses of these diseases are not completely understood, ${ }^{3,4}$ OCT has implicated anteroposterior tractional force as a reasonable cause. Moreover, it is unclear why patients with VMT develop different maculopathies. This question has been the focus of several recent studies. ${ }^{2,5-10}$

A major classification of the VMT syndrome has not yet been established and universally accepted. Based on the morphological findings of OCT, the pattern of vitreomacular adherence can be divided into two categories: V-shaped and J-shaped types. ${ }^{11,12}$ Another, distinct basis for classification (focal adhesion and broad adhesion $)^{9}$ is based on the concept that the diameter of the vitreomacular attachment is inversely related to macular morbidity and foveal deformation. The narrower the vitreomacular attachment is, the greater the force will be on the macula, whereas diffuse vitreomacular adhesion may distribute the
'Department of Ophthalmology, Federal University of São Paulo, UNIFESP, São Paulo, Brazil

Institut Universitari Barraquer, IUB, Universitat Autònoma de Barcelona, Barcelona, Spain

Correspondence: M Maia, Department of Ophthalmology, Federal University of São Paulo, UNIFESP, 821, 2nd floor, Botucatu Street, Vila Clementino, São Paulo, SP 04023-062, Brazil Tel: +55 11 50852042; Fax: +55 1150852003 . E-mail: maiamauricio@ terra.com.br

Received: 23 October 2013 Accepted in revised form: 28 April 2014 Published online: 4 July 2014 
tractional force beyond the border of the foveal region. ${ }^{7}$

The primary purpose of this study was to analyze the agreement between the classifications based on the morphology and the diameter of VMT syndrome. The secondary aim of the study was to correlate the morphological findings of VMT syndrome with specific maculopathies.

\section{Materials and methods}

The secondary data of patients diagnosed with VMT syndrome between January 2008 and January 2010 in the Institut Universitari Barraquer were reviewed after the approval of the ethical committee of Universitat Autònoma de Barcelona (UAB, Barcelona, Spain) and Federal University of São Paulo (UNIFESP, São Paulo, Brazil).

VMT syndrome was defined as an attachment of the posterior hyaloid to the macula with a surrounding vitreous detachment and associated tractional macular distortion and symptomatic loss of visual function. Each patient underwent complete clinical and ophthalmic examinations, including measurement of the bestcorrected visual acuity (BCVA), biomicroscopy, color fundus photography, and HD-OCT based on spectraldomain technology (Cirrus, Carl Zeiss Meditec, Dublin, CA, USA) with three-dimensional reconstruction. Pseudophakic patients who had CME were submitted to fluorescein angiography in order to compare with late Irvine-Gass syndrome, which generally exhibits significant capillary leakage, especially at the optic disc, on this examination. Otherwise, tractional CME, not linked with inflammatory aspects, shows only minimal leakage, if present at all. ${ }^{2}$

All eyes were divided according to two types of classification based on the relationship of the posterior hyaloid to the retina seen on OCT images. The first, related to the morphology of vitreofoveal adhesion, included two pathology groups: the V-shaped pattern (a perifoveal vitreous detachment with persistent vitreomacular adhesion) and the J-shaped pattern (an incomplete posterior vitreous detachment with persistent nasal attachment but detachment temporal to the fovea). The second classification was based on the diameter of the vitreomacular adhesion: focal VMT (maximal diameter of the vitreomacular attachment $\leq 1500 \mu \mathrm{m}$ ) and broad VMT (maximal diameter $>1500 \mu \mathrm{m}$ ) (Figure 1). The length of the vitreoretinal adhesion was measured using the macular cube $512 \times 128$ combo and an electronic caliper in the HD-OCT instrument. The horizontal extent of the vitreous attachment was determined by the maximal length of the vitreous adhesion in 128 horizontal B scan lines comprising 512 axial scans. To determine the vertical extent of the vitreous attachment, the number of horizontal scans in which vitreoretinal attachment was seen was multiplied by the space between the scans. The greatest length of the horizontal or the vertical extent was defined as the maximal diameter of the vitreomacular attachment. ${ }^{7}$ HD-OCT automatically measured the

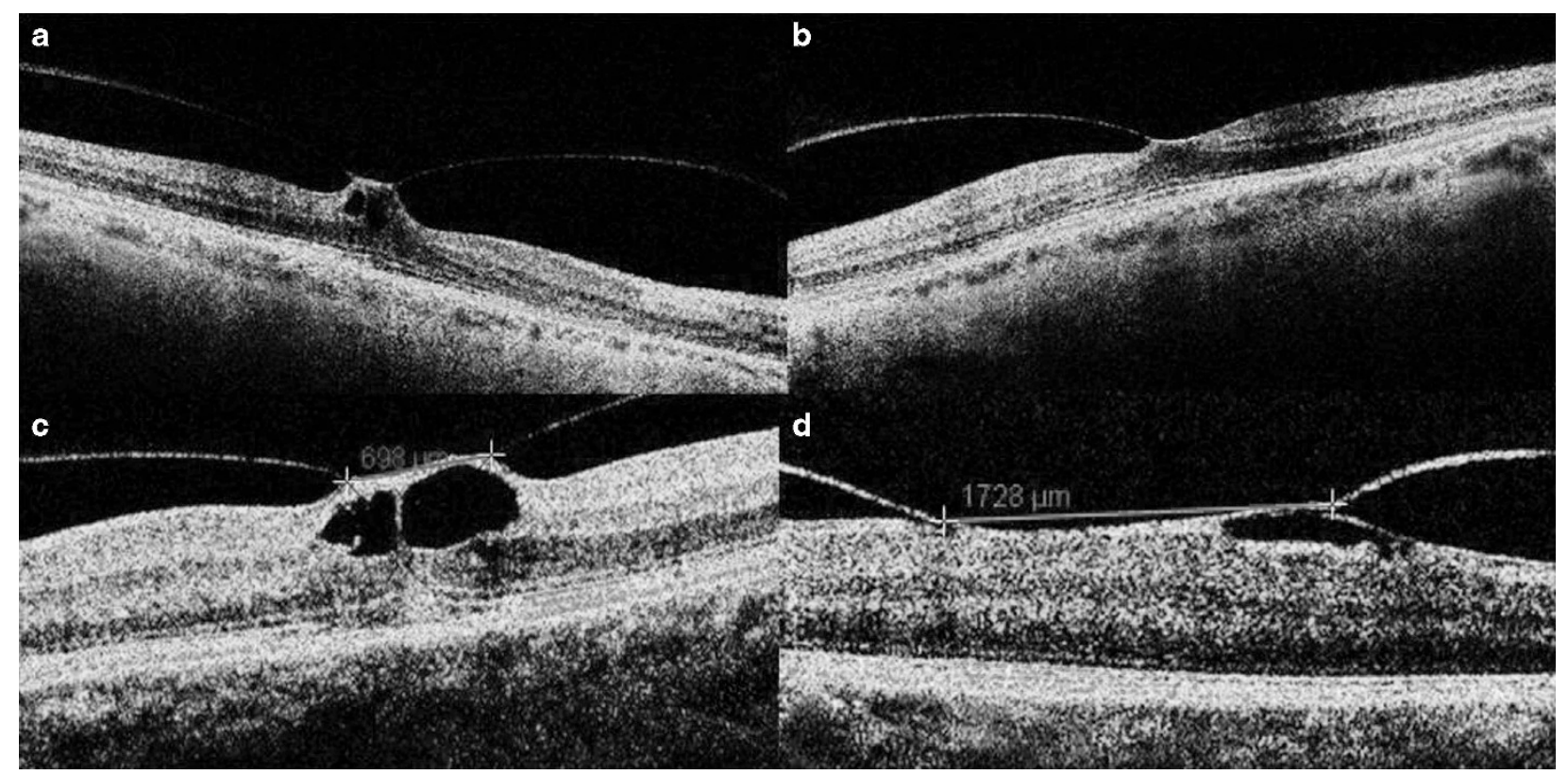

Figure 1 High-definition optical coherence tomography (OCT) images of vitreomacular traction (VMT) syndrome based on the pattern of vitreomacular adhesion: (a) V-shaped VMT and (b) J-shaped VMT. OCT images of the VMT syndrome classification based on the diameter of the vitreomacular adhesion: (c) focal VMT and (d) broad VMT. 
central macular thickness (CMT) in the central fovea. To compare the mean CMT between the subgroups, the eyes with an inner lamellar $\mathrm{MH}$, pseudohole, or full-thickness $\mathrm{MH}$ were excluded from the calculation, as proposed by Koizumi et al. ${ }^{7}$

The associations with some maculopathies, such as retinal thickening, foveal irregularity, retinoschisis, $\mathrm{MH}$, and subretinal detachment, were recorded. The presence of an ERM and tractional CME also was recorded and considered separately, since they often are associated with other maculopathies.

Statistical analysis was performed using software R.v.11.0 and SPSS v15.0. (SPSS Inc., Chicago, IL, USA). The variables considered were morphological classification of VMT (V-shaped or J-shaped); diameter classification of VMT (focal or diffuse); CMT (micron) and the maculopathies (absent or present): retinoschisis; retinal irregularity; $\mathrm{MH}$; subfoveal retinal detachment; ERM and CME. For statistical purposes, the decimal BCVA values were converted to the logarithm of the minimum angle of resolution (logMAR) visual acuity. The concordance between the classification proposals was analyzed using the kappa coefficient. The association between the maculopathies and the classifications was analyzed using the Fisher test and the relation between maculopathies and CMT was studied based on Mann-Whitney nonparametric test. For all analyses, $P<0.05$ was considered significant.

We certify that all applicable institutional and governmental regulations concerning the ethical use of human volunteers were followed during this research.

\section{Results}

Fifty-three patients (mean age, 63 years; range, 27-79 years) with the diagnosis of VMT syndrome were evaluated. Thirty patients were men (57\%). The mean duration of symptoms (low visual acuity and/or metamorphopsia) was 10 months.

The HD-OCT images showed V-shaped VMT in 29 cases and J-shaped VMT in 24 cases. Considering the same patients, 25 cases had focal VMT and 28 cases had broad VMT. High correlation was seen between V-shaped and focal VMT and between J-shaped VMT and broad VMT (kappa $=0.850 ; P<0.001$ ), except in four cases classified as V-shaped VMT and broad adhesions simultaneously (Table 1).

V-shaped VMT and focal VMT were highly correlated with prominent CME. Thirty-four eyes $(64.15 \%$ of all cases) had prominent CME. Twenty-one of them (84\% of focal cases) were associated with focal-based VMT while 13 patients ( $46.42 \%$ of broad cases) with broad-based VMT presented CME. MHs were also related to V-shaped and focal VMT (37.93\% and 44\%, respectively). All the
Table 1 Concordance between the classifications for VMT syndrome

\begin{tabular}{cccc}
\hline & \multicolumn{2}{c}{ Diameter classification } & Total \\
\cline { 2 - 3 } & Broad & Focal & \\
\hline $\begin{array}{c}\text { Morphological classification } \\
\text { J-shaped } \\
n\end{array}$ & 24 & & \\
$\quad \%$ & $45.3 \%$ & 0 & 24 \\
V-shaped & & $0 \%$ & $45.3 \%$ \\
$n$ & 4 & 25 & 29 \\
$\%$ & $7.5 \%$ & $47.2 \%$ & $54.7 \%$ \\
Total & & & \\
$n$ & 28 & 25 & 53 \\
$\%$ & $52.8 \%$ & $47.2 \%$ & $100,0 \%$ \\
\hline
\end{tabular}

Kappa $=0.850 ; P<0.001$.

Table 2 Association between maculopathies and classifications based on morphological and diameter of VMT syndrome

\begin{tabular}{|c|c|c|c|c|}
\hline Maculopathy & J-shaped & $\mathrm{P}$ & Broad VMT & $\mathrm{P}$ \\
\hline \multicolumn{5}{|l|}{ Retinoschisis } \\
\hline Absent & $21 / 50(42 \%)$ & 0.086 & $25 / 50(50 \%)$ & 0.238 \\
\hline Present & $3 / 3(100 \%)$ & & $3 / 3(100 \%)$ & \\
\hline \multicolumn{5}{|c|}{ Retinal irregularity } \\
\hline Absent & $9 / 32(28 \%)$ & 0.004 & $10 / 32(31 \%)$ & $<0.001$ \\
\hline Present & $15 / 21(71 \%)$ & & $18 / 21(86 \%)$ & \\
\hline \multicolumn{5}{|l|}{ Macular hole } \\
\hline Absent & $23 / 41(56 \%)$ & 0.007 & $27 / 41(66 \%)$ & 0.001 \\
\hline Present & $1 / 12(8 \%)$ & & $1 / 12(8 \%)$ & \\
\hline \multicolumn{5}{|c|}{ Subfoveal retinal detachment } \\
\hline Absent & $24 / 48(50 \%)$ & 0.056 & $27 / 48(56 \%)$ & 0.176 \\
\hline Present & $0 / 5(0 \%)$ & & $1 / 5(20 \%)$ & \\
\hline \multicolumn{5}{|c|}{ Epiretinal membrane } \\
\hline Absent & $2 / 23(9 \%)$ & $<0.001$ & $2 / 23(9 \%)$ & $<0.001$ \\
\hline Present & $22 / 30(73 \%)$ & & $26 / 30(87 \%)$ & \\
\hline \multicolumn{5}{|c|}{ Cystoid macular edema } \\
\hline Absent & $13 / 19(68 \%)$ & 0.020 & $15 / 19(79 \%)$ & 0.009 \\
\hline Present & $11 / 34(32 \%)$ & & $13 / 34(38 \%)$ & \\
\hline
\end{tabular}

Fisher test.

five cases of subfoveal detachment were found in V-shaped and focal VMT.

J-shaped VMT and broad VMT were associated with diffuse retinal thickening $(62.50 \%$ and $64.28 \%$, respectively). A hyperreflective linear signal on the macular surface, consistent with an ERM, was seen in 30 (56.6\%) patients. Among these 30 patients with ERM, 26 (92.85\% of the 28 broad cases) had broad-based VMT, and only 4 cases (16\% of the 25 focal cases) with focal VMT had an ERM (Table 2). 
Table 3 Comparison between patients with or without maculopathies and central macular thickness

\begin{tabular}{lcc}
\hline Maculopathies & Central macular thickness $(\mu \mathrm{m})$ & $\mathrm{P}$ \\
\hline $\begin{array}{l}\text { Retinoschisis } \\
\text { Absent }\end{array}$ & $414(202-697)$ & \\
Present & $543(335-634)$ & \\
Retinal irregularity & & \\
Absent & $385(262-655)$ & \\
Present & $468(202-697)$ & \\
Macular hole & & \\
Absent & & \\
present & $460(202-697)$ & \\
& $385(299-520)$ & \\
Subfoveal retinal detachment & & \\
Absent & $417(202-697)$ & \\
Present & $333(262-655)$ & \\
Epiretinal membrane & & 0.626 \\
Absent & & \\
Present & $377(202-655)$ & \\
Cystoid macular edema & $464(289-697)$ & \\
Absent & & \\
Present & $468(202-697)$ & \\
\hline
\end{tabular}

Mann-Whitney.

The mean CMT of all patients was $424 \pm 113 \mu \mathrm{m}$. It was slightly greater in cases with J-shaped or broad VMT (469 and $464 \mu \mathrm{m}$, respectively) than in those with $\mathrm{V}$-shaped or focal VMT (394 $\mu \mathrm{m}$ for both). These values did not differ significantly between morphological and diameter classification ( $P=0.129$ and 0.219 , respectively). The cases of VMT syndrome associated with ERM had a significantly thicker central macula $(464 \mu \mathrm{m})$ compared to those without an ERM (377 $\mu \mathrm{m} ; P=0.023)$ (Table 3).

Patients with focal adhesions had a lower BCVA (0.37 decimal BCVA; $0.50 \log$ MAR BCVA) than those with broad adhesions (0.44 decimal BCVA; 0.42 logMAR). However, it was not statistically significant $(P=0.198)$. Patients classified as V-shaped (0.41 decimal BCVA; 0.45 logMAR BCVA) or J-shaped (0.40 decimal BCVA; 0.46 logMAR BCVA) had similar BCVA $(P=0.816)$.

\section{Discussion}

The VMT syndrome has been described as having distinct anatomic presentations. Each configuration has unique implications regarding the anatomic and functional outcomes, which has led to some proposals for classifications based on the VMT pattern on OCT. $5,7,11,12$ The classic VMT morphological features of incomplete V- and J-shaped VMT have been described previously, with better outcomes associated with the V-shaped configuration. ${ }^{11,12}$ Another classification was postulated based on the concept that the diameter of the vitreal attachment is inversely proportional to the macular morbidity. ${ }^{9}$ According to this physical correlation, the VMT syndrome can be divided into two categories: focal $(\leq 1500 \mu \mathrm{m})$ and broad $(>1500 \mu \mathrm{m}){ }^{5,7}$ Some authors have even proposed that the focal type may be a separate syndrome, that is, the vitreofoveal traction syndrome, ${ }^{6,13}$ with a foveal adhesion size $<500 \mu \mathrm{m}$. Accordingly, it is believed that classic VMT syndrome is associated with a macular adhesion size of $\sim \geq 1500 \mu \mathrm{m}$ (between 1500 and $6000 \mu \mathrm{m})$. Most authors consider focal VMT to be a form of VMT syndrome closely related to tractional CME and $\mathrm{MH}$ formation. 2,5,7,9,11,12 In the current study, all 53 cases of VMT syndrome were classified as both V- or J-shaped and the focal or broad type. To the best of our knowledge, this study is unique in the comparison of the different classifications of VMT syndrome.

A high correlation was observed between focal and V-shaped type and between broad and J-shaped type, except in four cases, which had broad adhesion despite the presence of a V-shaped pattern (Figure 2). These four cases had common characteristics and developed in a manner similar to those with broad vitreal attachment regarding associated maculopathies and visual function. Furthermore, these four eyes were associated with ERM and had a thick and irregular macular surface, also related to broad cases. Although both classifications are highly coincident, the discordant cases behave in a manner similar to the VMT syndrome patients classified based on the diameter of adhesion. Therefore, we hypothesized that the classification of VMT syndrome based on diameter and not the pattern of adhesion might better reflect the related anatomic lesions and visual function.

Specific macular entities were associated with a particular VMT type. Among eyes with focal and V-shaped VMT, CME was the most frequent macular change, followed by impending or full-thickness MHs and subfoveal detachment. Otherwise, among cases with broad and J-shaped VMT the diffuse retinal thickening largely associated with ERM prevailed, while only one case had $\mathrm{MH}$. These results suggested that certain types of adhesions are more prevalent with specific disease processes, mainly ERM, MH, and CME. Recent studies have reported strong correlations between ERM and the VMT syndrome, which may play a role in chronic VMT syndrome. ${ }^{5,7}$ In the current study, the presence of an ERM was associated with broad VMT. ERM may increase the strength of the residual vitreomacular adhesion, and the VMT stress can be prolonged by preventing spontaneous separation of the vitreous from the macula. 2,5,7 In addition, a vitreomacular adhesion concomitant with ERM resulted in a significantly thicker central macula, which could have been due to the contractile forces of the ERM that increased the tangential traction beyond the anteroposterior traction 


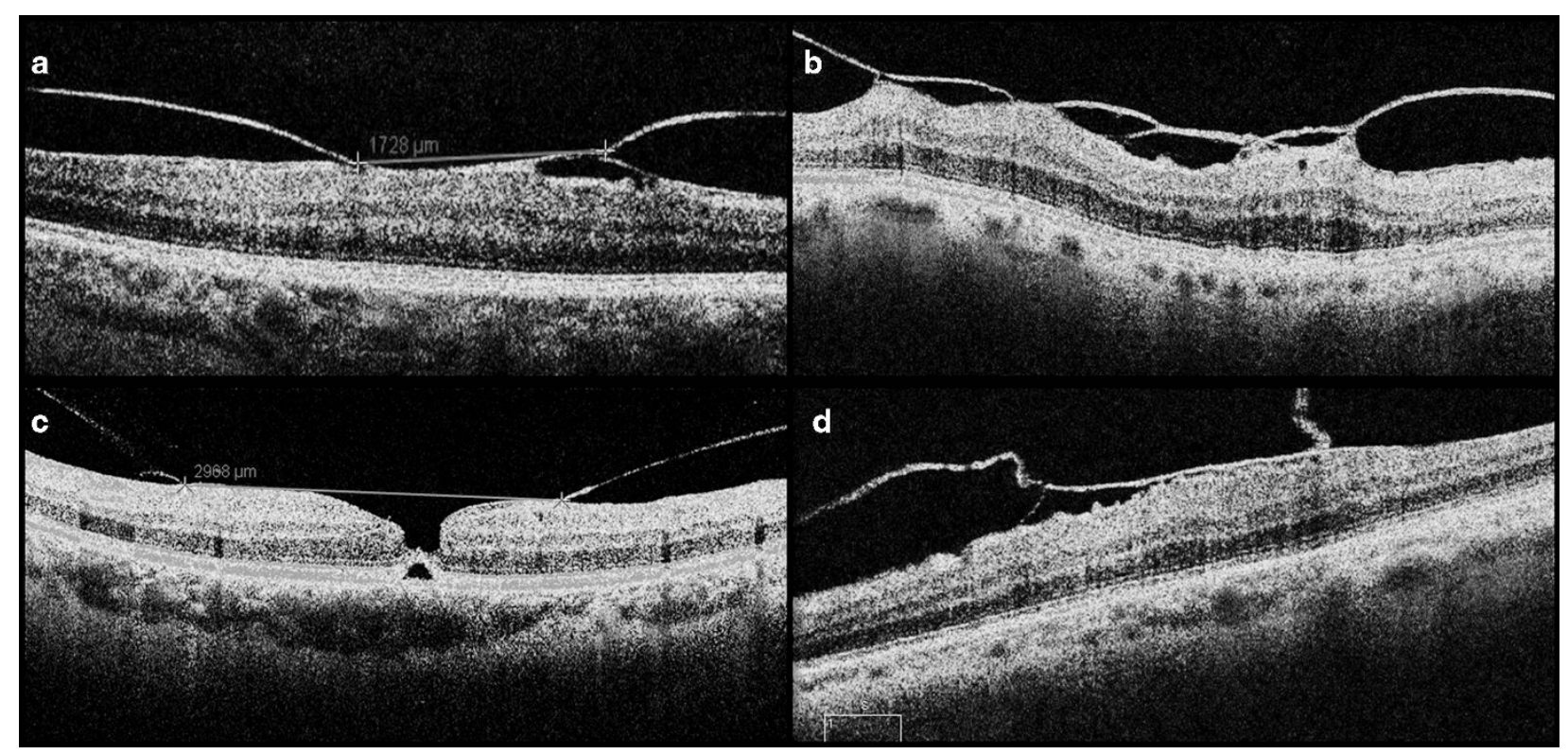

Figure 2 High-definition optical coherence tomography images of patients classified as broad type, based on diameter of the vitreomacular adhesion, and V-shaped, based on the pattern of the vitreomacular adhesion (a-d).

caused by the VMT.7,14 The vitreoretinal relationship in eyes with tractional CME is similar to that seen in eyes with an early-stage idiopathic $\mathrm{MH}^{2}$ The smaller the area of the foveal attachments, the greater the force that is exerted, causing more serious disease. ${ }^{9}$ This condition of focal adhesion, also known as vitreofoveal traction syndrome, implies a $\mathrm{MH}$ and $\mathrm{CME}$ formation. ${ }^{15-18}$ In the current study, the presence of $\mathrm{MH}$ was correlated with a V-shaped and focal VMT, besides other anatomic lesions associated with strong and punctual anteroposterior traction, such as subfoveal detachments. The strong effect of narrow bands of adhesion could lead to a variant of VMT, also referred as tractional CME. In the present study, prominent CME was found in $84 \%$ of focal-based VMT while only $46.42 \%$ of broad cases had this macular change, supporting this assumption. ${ }^{2}$ The reason that vitreofoveal traction leads to $\mathrm{MH}$ in some cases, while tractional CME without hole formation develops in other cases, even with time, has yet to be determined. ${ }^{2}$

In conclusion, although highly concordant, the classification based on the diameter of the adhesion (focal or broad, measured in $\mu \mathrm{m}$ ) and not on the adhesion morphology (V- or J-shaped) reflected more accurately the specific macular changes. We recommend that the classification based on the diameter of the adhesion instead of the morphology should be used in current practice. V-shaped and focal VMT led to tractional CME and $\mathrm{MH}$ while J-shaped and broad VMT were related to ERM and diffuse retinal thickening. The size and strength of the residual VMT may define the specific associated maculopathy. Future studies are necessary to define if classification based on the diameter of the adhesion may better reflect the prognosis of VMT, and to understand the specific associated maculopathies.

\section{Summary}

What was known before

- Vitreomacular traction (VMT) syndrome was considered an unusual macular condition and unrelated to other macular diseases.

- It was unclear why patients with VMT syndrome develop different maculopathies.

- Although the morphological classification (V- or J-shaped) has been worldwide used, a major classification of the VMT syndrome has not yet been established and universally accepted.

What this study adds

- The VMT syndrome contributes to a wide spectrum of macular diseases, including cystoid macular edema (CME), macular holes (MHs), and epiretinal membranes (ERMs).

- V-shaped and focal VMT lead to tractional CME and MHs, while J-shaped and broad VMT is related to ERM and diffuse retinal thickening. The size and strength of the residual VMT may define the specific associated maculopathy.

- Although highly concordant, the classification based on the diameter of the adhesion (focal or broad) and not on the adhesion morphology (V- or J-shaped) reflected more accurately the specific macular changes. We recommend that the classification based on the diameter of the adhesion instead of the morphology be used in current practice.

\section{Conflict of interest}

The authors declare no conflict of interest. 


\section{Acknowledgements}

We are grateful to our colleagues in the Vitreoretinal Service Unit of Federal University of São Paulo and Instituto Universitário Barraquer; Fundación Carolina, Spain; Fundação de Amparo à Pesquisa do Estado de São Paulo and CNPq (Conselho Nacional de Pesquisa). This study was the winner of Joaquín Rutllán 2010 Award, from the Instituto Barraquer de Oftalmología, Barcelona, Spain.

\section{References}

1 McDonald HR, Johnson RN, Schatz H. Surgical results in the vitreomacular traction syndrome. Ophthalmology 1994; 101: 1397-1402; discussion 1403.

2 Johnson MW. Tractional cystoid macular edema: a subtle variant of the vitreomacular traction syndrome. Am J Ophthalmol 2005; 140: 184-192.

3 Smiddy WE, Michels RG, Green WR. Morphology, pathology, and surgery of idiopathic vitreoretinal macular disorders. A review. Retina 1990; 10: 288-296.

4 Shechtman DL, Dunbar MT. The expanding spectrum of vitreomacular traction. Optometry 2009; 80: 681-687.

5 Chang LK, Fine HF, Spaide RF, Koizumi H, Grossniklaus HE. Ultrastructural correlation of spectral-domain optical coherence tomographic findings in vitreomacular traction syndrome. Am J Ophthalmol 2008; 146: 121-127.

6 Johnson MW. Posterior vitreous detachment: evolution and complications of its early stages. Am J Ophthalmol 2010; 149: 371-382 e1.

7 Koizumi H, Spaide RF, Fisher YL, Freund KB, Klancnik Jr JM, Yannuzzi LA. Three-dimensional evaluation of vitreomacular traction and epiretinal membrane using spectral-domain optical coherence tomography. Am J Ophthalmol 2008; 145: 509-517.
8 Mirza RG, Johnson MW, Jampol LM. Optical coherence tomography use in evaluation of the vitreoretinal interface: a review. Surv Ophthalmol 2007; 52: 397-421.

9 Spaide RF, Wong D, Fisher Y, Goldbaum M. Correlation of vitreous attachment and foveal deformation in early macular hole states. Am J Ophthalmol 2002; 133: 226-229.

10 Jackson TL, Nicod E, Simpson A, Angelis A, Grimaccia F, Kanavos P. Symptomatic vitreomacular adhesion. Retina 2013; 33(8): 1503-1511.

11 Sonmez K, Capone Jr A, Trese MT, Williams GA. Vitreomacular traction syndrome: impact of anatomical configuration on anatomical and visual outcomes. Retina 2008; 28: 1207-1214.

12 Yamada N, Kishi S. Tomographic features and surgical outcomes of vitreomacular traction syndrome. Am J Ophthalmol 2005; 139: 112-117.

13 Smiddy WE, Flynn Jr HW. Pathogenesis of macular holes and therapeutic implications. Am J Ophthalmol 2004; 137: 525-537.

14 Odrobina D, Michalewska Z, Michalewski J, Dziegielewski K, Nawrocki J. Long-term evaluation of vitreomacular traction disorder in spectral-domain optical coherence tomography. Retina 2011; 31: 324-331.

15 Gaudric A, Haouchine B, Massin P, Paques M, Blain P, Erginay A. Macular hole formation: new data provided by optical coherence tomography. Arch Ophthalmol 1999; 117: 744-751.

16 Haouchine B, Massin P, Gaudric A. Foveal pseudocyst as the first step in macular hole formation: a prospective study by optical coherence tomography. Ophthalmology 2001; 108: $15-22$.

17 Hikichi T, Akiba J, Trempe CL. Effect of the vitreous on the prognosis of full-thickness idiopathic macular hole. Am J Ophthalmol 1993; 116: 273-278.

18 Ito $\mathrm{Y}$, Terasaki $\mathrm{H}$, Suzuki T et al. Mapping posterior vitreous detachment by optical coherence tomography in eyes with idiopathic macular hole. Am J Ophthalmol 2003; 135: 351-355. 